\title{
Application On the Damage Prediction of Prestressed and Stayed Cable In the Bridge Based Hilbert-Huang Transform
}

\author{
Xiang $\mathrm{LI}^{1, \mathrm{a}}$, Nianchun DENG ${ }^{2, \mathrm{~b}}$ \\ ${ }^{1}$ Department of Electrical Engineering, Guangxi Technological College of Machinery and Electricity, \\ Nanning, 530007, China \\ ${ }^{2}$ Department of Civil Engineering, Guangxi University, Nanning, 530004, China \\ aemail: xiangzi21th@hotmail.com, bemail: dengnch@163.com
}

Keywords: Hilbert-Huang Transform; Empirical Mode Decomposition; Flexural Wave

\begin{abstract}
In the industry, the damage detection of stayed cable is an important research subject. A proper method for the vibration signal processing is indispensable. The Hilbert-Huang Transform (HHT), which has been successfully applied to many different fields in the last ten years, may provide a promising method for this purpose. The purpose of this research is to detect the crack on beams by HHT of transient flexural waves. The mean values of absolute relative error are all less than $2.5 \%$. In addition, the characteristics on Hilbert spectrum of the damage size were also studied. This study may contribute to the damage size estimation in the near future.
\end{abstract}

\section{Introduction}

Important large-scale structure of life and property is at stake is enormous, once lax inspection and maintenance, this time not only caused economic losses, but also endanger personal safety, irreversible regret occur. The most common method used to analyze the signal Fourier transform [1] and wavelet conversion [2], but both are based on a preset function to analyze the signal substrate, and analysis are all linear operation, and it is only applicable to linear smooth physical phenomena. But real-life phenomenon mostly nonlinear and non-stationary, Hilbert-Huang Transform (HHT) [3] not established a base to decompose the signal, but the use of a time-varying signal itself time scale to raise the base. Since the substrate is generated by the signal, and therefore have a physical characteristic of the signal, smooth the signal is not limited. This base has the physical characteristics of the signal deemed one of the component with the signal, will be aware of instantaneous frequency and instantaneous amplitude of each moment. Therefore HHT lasted for non-stationary signals have better resolution.

\section{Theoretical Basis of HHT}

Instantaneous frequency is generally defined as the phase differential, but the nature of the signals are below real signals, you can not clearly stated signal phase and amplitude, therefore, must be a real signal to the plural form, it said it can be instantaneous frequency seeking real signal out. If the signal is a real number, which corresponds to the complex signal (complex signal) is

$$
Z(t)=S_{r}(t)+j S_{i}(t)=A(t) e^{j \varphi(t)}
$$

In the type:

$$
\left\{\begin{array}{c}
A(t)=\sqrt{S_{r}^{2}(t)+S_{i}^{2}(t)} \\
\varphi(t)=\tan ^{-1}\left(S_{i}(t) / S_{r}(t)\right)
\end{array}\right.
$$

You can get instantaneous frequency

$\omega_{i}(t)=\varphi^{\prime}(t)$

Analytical signal is used to indicate a real signal (Analytic Signal). The imaginary term analytic signal is Hilbert-Huang Transform real signal is defined as 


$$
H[s(t)]=\frac{1}{\pi} \int_{-\infty}^{+\infty} \frac{s\left(t^{\prime}\right)}{t-t^{\prime}} d t^{\prime}=s(t) * \frac{1}{\pi \bullet t}
$$

That is the original signal and the convolution integral (Convolution Integral), therefore, the local characteristics of Hilbert-Huang Transform can be analyzed.

Thus, for a real signal to obtain an imaginary term analytic signal by Hilbert-Huang Transform, and then by the formula (1), (2) and (3) can be obtained instantaneous frequency. However, if there is no signal at any time limit, the calculated instantaneous frequency may not make sense [4] [5]. For the average value of zero signal, the instantaneous frequency is defined only has the physical meaning. In order to obtain the correct instantaneous frequency, it requires certain restrictions.

\section{Hilbert-Huang Transform}

Hilbert-Huang Transform consists of two main steps[6] [7]: Empirical Mode Decomposition (EMD) and Hilbert transform (HT). One of the most critical part is the information EMD decomposition method, EMD can be based on the time scale of the signal itself (characteristic time scale) signals from high-frequency to low frequency divided into a group of several (usually small number of) basis functions, also known as the essence mode function (Intrinsic Mode Functions, IMFs) and a trend term (trend). Hilbert transform can sensitively capture the local phase changes in the IMF, to strike a physically meaningful instantaneous frequency, the establishment - Frequency energy, three-dimensional maps that Hilbert Spectrum. But for other non-IMF time series would have no effect. According to Hilbert Spectrum characteristics, define marginal spectrum (Marginal Spectrum). Because of Hilbert-Huang Transform can understand the distribution of the instantaneous frequency and energy, while the IMF base function with a variety of signal that varies with an adaptive (adaptive), therefore, EMD can analyze nonlinear (Non-linear) or steady (Non-stationary) time series behavior.

IMF set up to meet the Hilbert transform instantaneous frequency for restrictions adopted pre-processing stage. ENID is the signal into a combination of IMF while IMF meet each had two conditions mentioned earlier. In fact, EMD effect by repeated screening program (Shifting Process) to gradually find the IMF. Process screening procedures are as follows:

1, first find out all the local maxima and local minima, and then use cubic spline function (cubic spline) will be chained to the local maxima of the envelope, the same will be the local minimum values in series into the lower envelope;

2, find the average of the upper and lower envelope to obtain the mean envelope;

3 , the original signal and subtract the average envelope, get the first component.

$h_{1}(t)=s(t)-m_{1}(t)$

This completes the first screening program, and then check compliance with IMF conditions, if not, return to step 1 , and will begin to run a second screening process as the original signal. That

$h_{2}(t)=h_{1}(t)-m_{2}(t)$

Repeat screening times,

$h_{k}(t)=h_{k-1}(t)-m_{k}(t)$

Until the resulting component meets the definition of IMF is reached.

\section{Flexural Wave Theory}

Consider the infinitely long Timshenko passed on [8] Prestressed Cable sine wave (Harmonic Waves). There are two ways to obtain the dispersion equation (Dispersion Equation).

1 , assumed external force is 0 , and

$y=B_{1} e^{i(\gamma x-\omega t)}, \psi=B_{2} e^{i(\gamma-\omega t)}$

In the type(8): $\gamma$ is wave number, $\omega$ is angular frequency, and taken the formula (8) into the equation of motion Timoshenko beam, obtained two equations Homogeneous 
$\left(G A \kappa \gamma^{2}-\rho A \omega^{2}\right) B_{1}+i G A \kappa \gamma B_{2}=0$

$i G A \kappa \gamma B_{1}-\left(G A \kappa+E I \gamma^{2}-\rho I \omega^{2}\right) B_{2}=0$

Then

$$
\frac{B_{1}}{B_{2}}=i \frac{G A \kappa \gamma}{\left(G A \kappa+E I \gamma^{2}-\rho I \omega^{2}\right)}
$$

2, taken the motion equation of Timoshenko beam differential, then

$$
G A \kappa\left(\frac{\partial^{2} y}{\partial x^{2}}-\frac{\partial \psi}{\partial x}\right)+E I \frac{\partial^{3} \psi}{\partial x^{3}}=\rho I \frac{\partial^{3} \psi}{\partial t^{2} \partial x}
$$

Assumed $q=0$, then

$$
\frac{\partial \psi}{\partial x}=\frac{\partial^{2} \psi}{\partial x^{2}}-\frac{\rho}{G \kappa} \frac{\partial^{2} y}{\partial t^{2}}
$$

Push export substitution Timoshenko beam equations of motion, assuming sine wave solutions for the transfer, you can get into formula (11) the dispersion equation.

The phase velocity and frequency of the relationship into formula (12), The group velocity may be defined as, so

$$
\bar{c}_{g}=\frac{c_{g}}{c_{s}}=\frac{\bar{c}^{2}\left(1+\frac{E}{G \kappa}\right)-2 \frac{E}{G \kappa}}{\bar{c}\left[\bar{c}^{2}\left(2-\frac{1}{\bar{\omega}^{2}}\right)-\left(1+\frac{E}{G \kappa}\right)\right]}
$$

By the formula (13) shows the velocity as a function of frequency, this is bending wave dispersion phenomena (dispersion), so you can use this feature to find the location of the fracture of the beam. The value of the cross-sectional shape respectively is relevant, square and circular cross-section.

\section{Experimental Equipment and Systems Architecture}

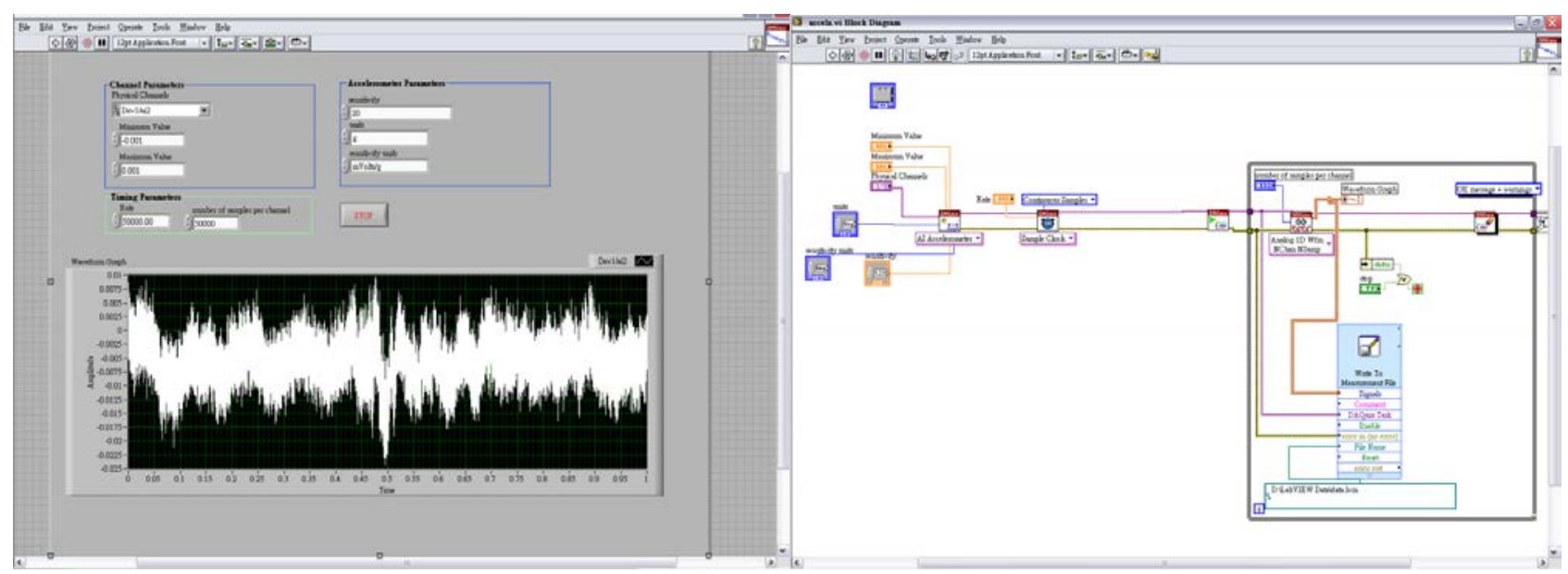

a) The instrument panel

b) Block Diagram

Fig. 1 The vibration measurement virtual instrument

You can choose to write measurement software measurement Labview virtual instrument vibration signals, as shown in Figure 1 . So you can get acceleration lasted bending wave signal on the cable prestressing, when Hilbert-Huang Transform can be obtained by frequency diagram shown in Figure 2, Figure 3. Bending waves are dispersive waves at different frequencies have different phase and group velocity can be used to bending wave transmission and reflection time on the prestressing cables, as well as the travel distance in the prestressing cables, perform inverse velocity. When the observable frequency graph, you can find many peaks exist, which is under the impact of signal tapping, bending wave is transmitted to the first prestressed cables reflected back to the other end of the border accelerometer measured signal, for the second reflection, and so on. 
Thus, group velocity estimation formula

$$
c_{g}(f)=\frac{2 L}{t_{k+1}(f)-t_{k}(f)}, k=0,1,2, \cdots
$$

In the type: $L$ prestressed cable length.

If there are defects, shown in Figure 4, when the wave passes to the defect will be part of the energy is reflected back to pass, part of the energy to pass on prestressed cables. Therefore, when the frequency chart, between the peak and the first peak shock wave boundary reflection, there will be a peak defect reflected waves can be obtained and defects occur in the shock wave at the time difference between the return wave emission, the use of theoretical group velocity value, can be obtained from the defect between the Department and the accelerometer.

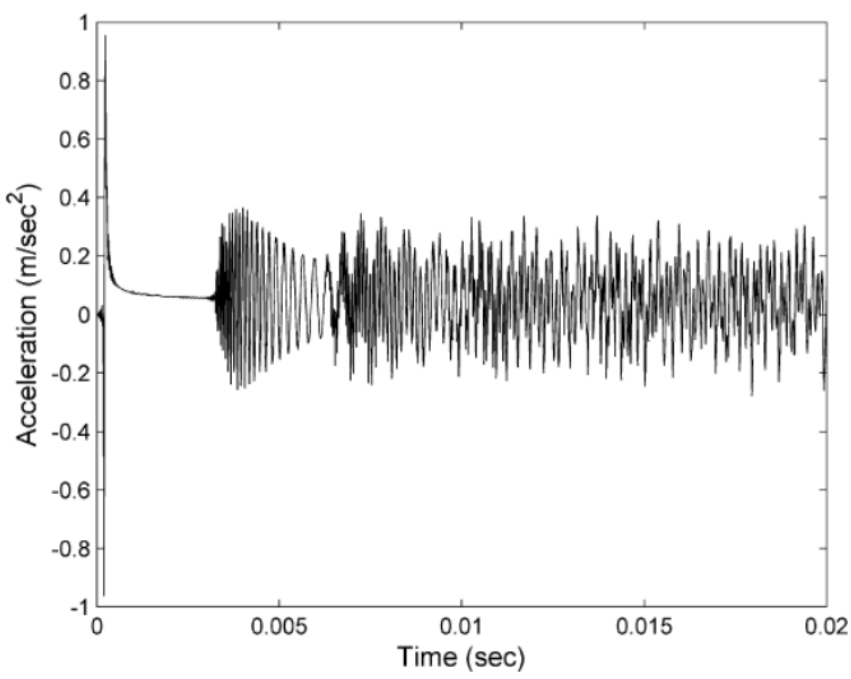

Fig. 2 Acceleration lasted signal diagram

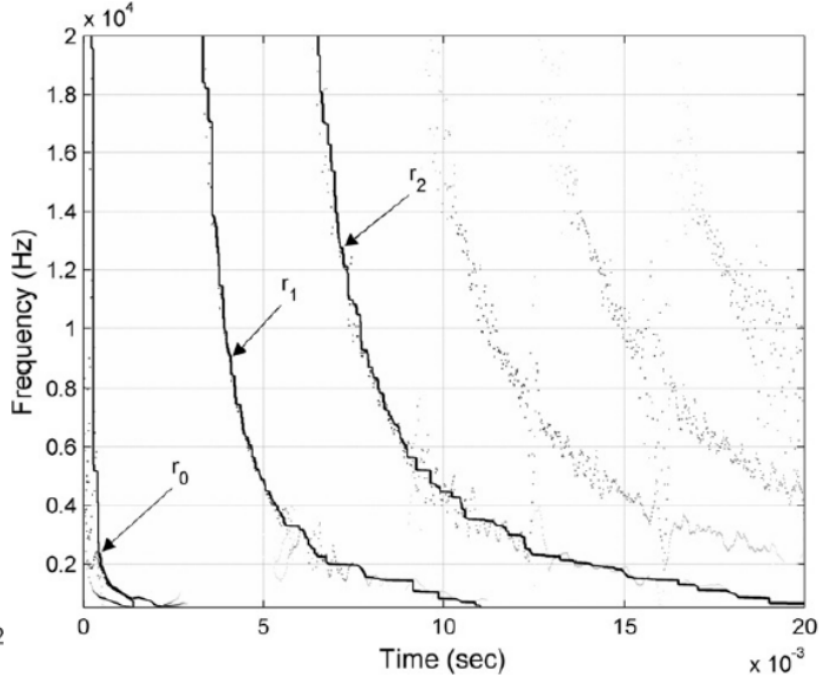

Fig. 3 The frequency diagram in HHT

$$
d=\frac{c_{g}(f) *\left[t_{c}(f)-t_{0}(f)\right]}{2}
$$

In the type: $t_{c}$ is peak time of defects reflection , $t_{0}$ is peak time of the shock wave.

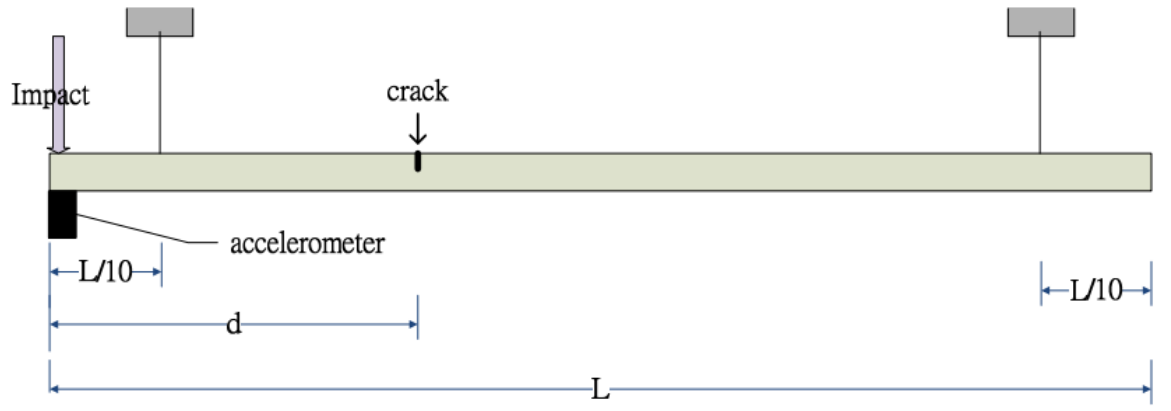

Fig.4 Schematic defect on the cable

\section{Signal Analysis and Processing}

Measurement software can select virtual instrument Labview prepared to measure vibration signal to write signal analysis program HHT use Matlab, to simulate the prestressed cables with an elongated circular beam, No. 304 stainless steel material selection, size of $2700 \mathrm{~mm} \times \Phi 10 \mathrm{~mm}$, measuring the acceleration signal analysis, experimental take sampling frequency is $50 \mathrm{KHz}$, add white noise on the order of, plus a total of 50 times the average number.

In order to compare the experimental and theoretical velocity measured velocity difference on different frequencies, the definition of absolute relative error ARE (Absolute Relative Error)

$$
\operatorname{ARE}(f)=\frac{\left|v_{I}(f)-c_{g}^{T}(f)\right|}{c_{g}^{T}(f)}, \quad i=1,2, \cdots
$$


In the type(16): $i$ is the group velocity computed by different wave peaks value; $T$ is Timoshenko Model.

There are 100 sampling points before the time point and the 1900 sampling points after it to be taken for analysis, which maximum acceleration occur, Figure 5 is an accelerometer measured acceleration of the original signal, after Figure 6 decomposed IMF and the residual value, Figure 7 as Hilbert Spectrum. Figure 8 will do when the frequency peak graph curve fitting, and then seek respectively, by the time difference between the group velocity and wave transmission distance, as shown in Figure 9, Figure 10 is the actual velocity and velocity error theory.

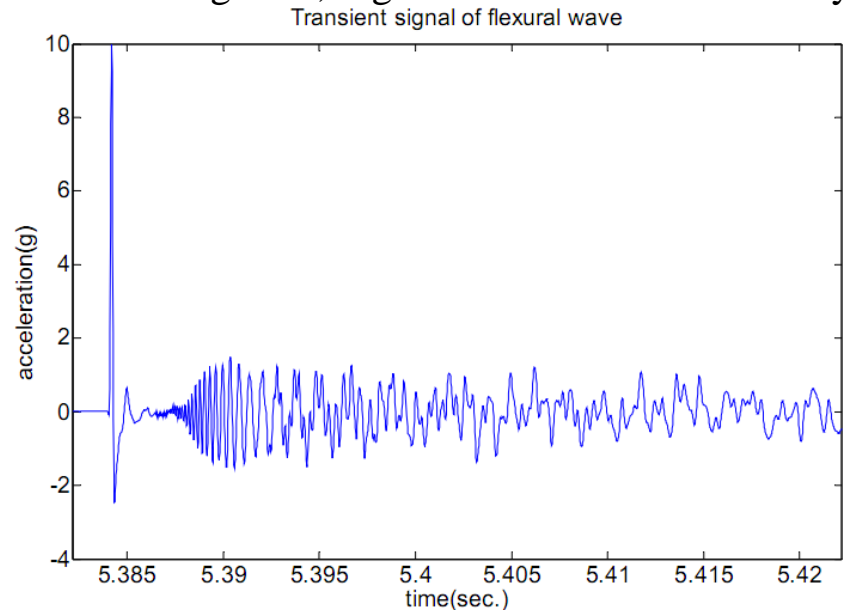

Fig.5 Acceleration signal

Hilbert-Huang spectrum

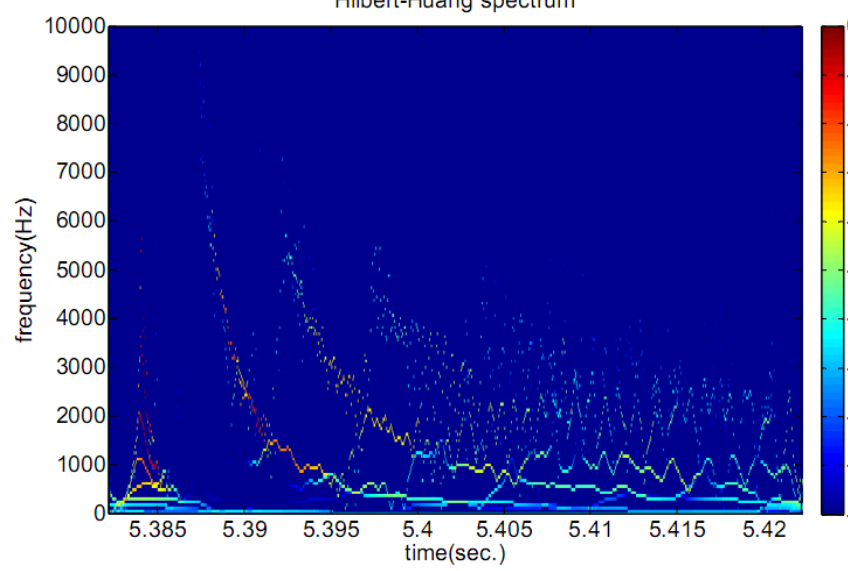

Fig.7 Hilbert Spectrum in time

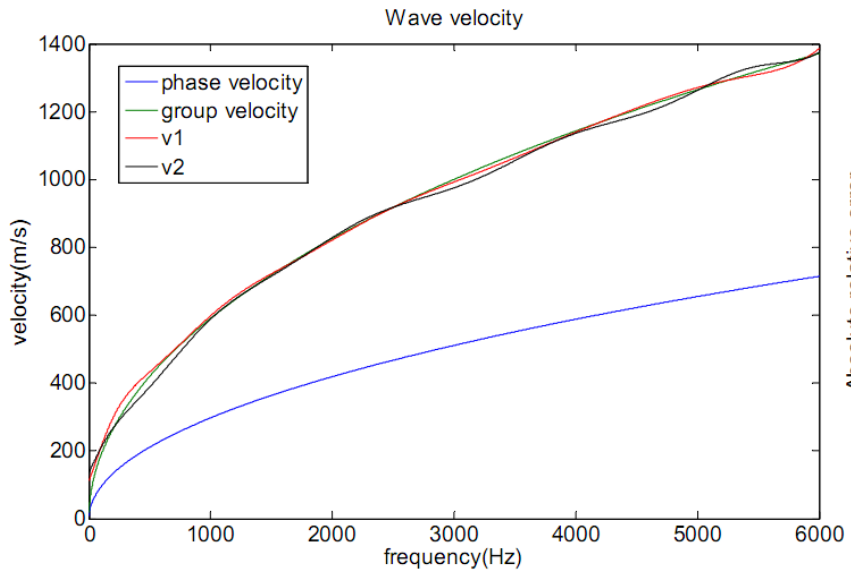

Fig. 9 velocity comparing

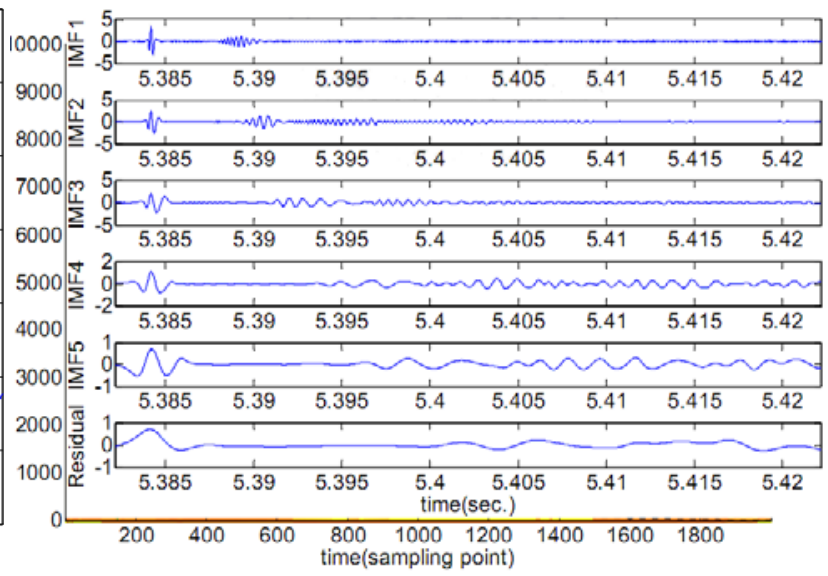

Fig. 6 IMF

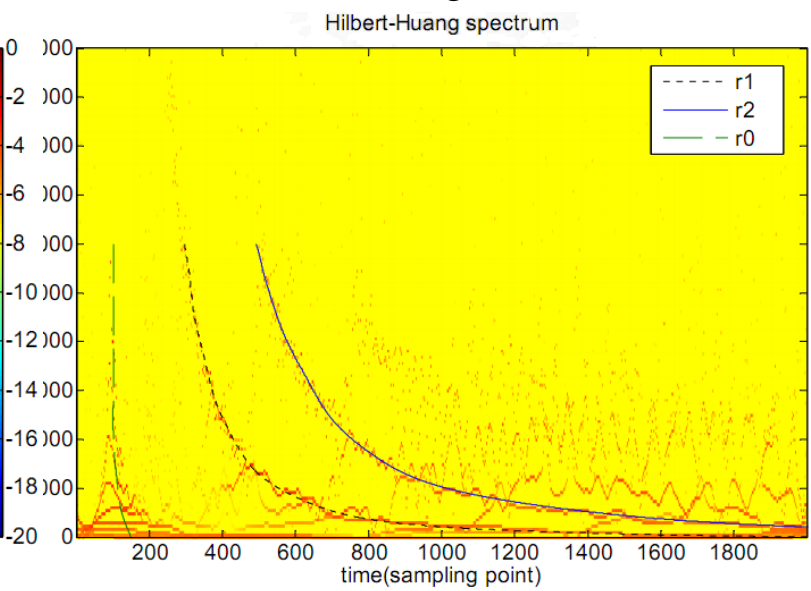

Fig.8 Hilbert Spectrum in frequency

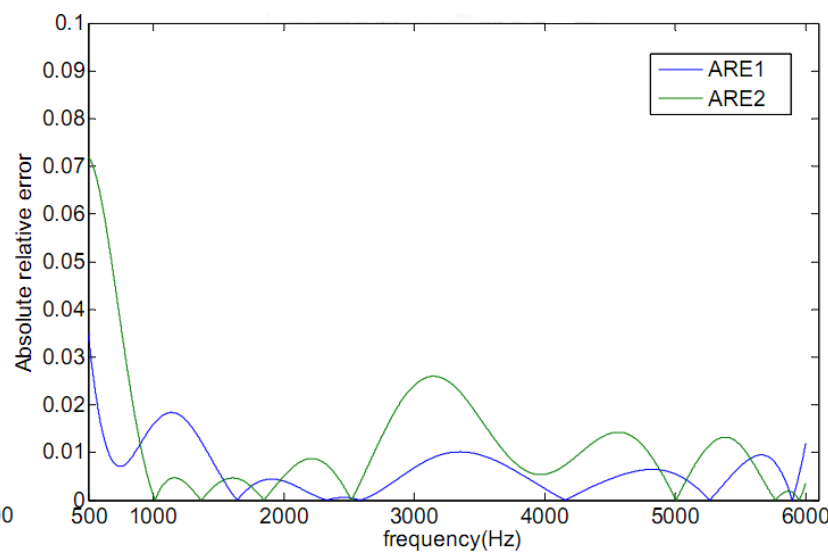

Fig.10 velocity velocity error statistics

From the experimental results, the Hilbert Spectrum can clearly see the three peaks, namely, by the first and second peaks reflected wave reflected wave peak group may seek speeds. From the figure, at a low frequency error is larger than the high frequency error may be due to poor low-resolution, low-frequency portion of the signal is low energy, low-frequency noise outside 
interference. The mean error of the mean error relatively large, because the shock wave, its peak curve after curve fitting than the reflected wave peak curve accurate, overall, error velocity experiments and theoretical values obtained, both in high frequency or low frequency is not large, and the error does not exceed $2.5 \%$ of the average, so that the method can be used in this report to the elongated beam bending wave group velocity estimation is feasible.

\section{Conclusion}

It is feasible to above method for elongated beam bending wave group velocity estimation. Compare IMF3 and IMF2 defects or boundary reflected wave reflected wave arrival time, are the higher frequency of the reflected wave signal IMF2 occurs first, in line with bending wave dispersion phenomena. By comparison, the peak can be confirmed as a defect between the reflected wave after reflection and boundary reflection because of defects caused by overly complex waveforms confusion.

\section{Acknowledgement}

In this paper, the research was sponsored by Guangxi Colleges and Universities Talents Scheme ( Project No. 40, Gui-Jiao-Ren [2011]).

\section{References:}

[1] A. H. Nuttall. On the Quadrature Approximation to the Hilbert Transform of Modulated Signals [C], Proc. IEEE, 1966, 54: 1458-1459.

[2] N. E. Huang, Z. Wu. A Review on Hilbert-Huang Transform: Method and its Applications to Geophysical Studies [J], Reviews of Geophysics, 2008, 46: 1-23.

[3] Z. Wu, N. E. Huang. Ensemble empirical mode decomposition: A noise-assisted data analysis method[A], Center for Ocean-Land-Atmosphere Studies, Technical Report series, 2005, 193(173): 1156-1187.

[4] Y. N. Jeng. The Moving Least Squares and Least p-Power Methods for Random Data[C], Kenting, Taiwan: The 7th National Computation Fluid Dynamics Conference, Aug. 2000: 9-14.

[5] Y. N. Jeng, G. P. G. Huang, H. Chen. Wave Decomposition in Physical Space Using Iterative Moving Least Squares Methods[C], Tai-Tung, Taiwan: The 11th National Computation Fluid Dynamics Conference, Aug. 2004: 23-29.

[6] X. Xiong, Y. X.i Chen. Remote Monitoring Based on LabVIEW Scenery Complementary Power Supply[J], Foreign Electronic Measurement Technology, 2012,31(9): 34-36. (in Chinese)

[7] Y. L. Guo, R. Tian, Y. S. He. Bridge Structural Health Monitoring Project Early Warning System[J], Building Technology, 2012,43(2): 125-127. (in Chinese)

[8] Nesterenko B G, Nesterenko G I. A Way to Secure Operational Safety for an Aircraft Structure According to a Strength Criterion[J]. Journal of Machinery Manufacture and Reliability, 2013, 42(1): 62-75. (in Chinese) 